\title{
Giant monopole resonance in transitional and deformed nuclei
}

\author{
U. Garg \\ Physics Department, University of Notre Dame, Notre Dame, Indiana 46556* \\ and Cyclotron Institute, Texas A\&M University, College Station, Texas 77843 \\ P. Bogucki, J. D. Bronson, Y.-W. Lui, and D. H. Youngblood \\ Cyclotron Institute, Texas A\&M University, College Station, Texas 77843
}

(Received 30 June 1983)

\begin{abstract}
Small-angle inelastic $\alpha$-scattering measurements have been made at $E_{\alpha}=129 \mathrm{MeV}$ on ${ }^{144,148} \mathrm{Sm}$ and ${ }^{142,146,150} \mathrm{Nd}$ to investigate the giant monopole resonance in transitional and deformed nuclei. The experimental data reveal a mixing of $L=0$ and $L=2$ modes in ${ }^{148} \mathrm{Sm}$ resulting in almost identical angular distributions for the two components of the giant resonance peaks in the angular range $2^{\circ}-6^{\circ}$. A "splitting" of the giant monopole resonance is observed in ${ }^{150} \mathrm{Nd}$; the extent of this splitting is smaller than that reported for ${ }^{154} \mathrm{Sm}$. Comparison is made with the predictions of various theoretical models.
\end{abstract}

$\left[\begin{array}{c}\text { NUCLEAR REACTIONS }{ }^{144,148} \mathrm{Sm},{ }^{142,146,150} \mathrm{Nd}\left(\alpha, \alpha^{\prime}\right), E_{\alpha}=129 \mathrm{MeV} . \text { Mea- } \\ \text { sured } E_{x}, \sigma(\theta) \text {, giant resonances; deduced } L \text {, mixing of modes. }\end{array}\right]$

\section{INTRODUCTION}

The effect of deformation on the giant resonances (GR's) has been investigated in some detail in the past few years. In particular, the splitting of the giant monopole resonance $(\mathrm{GMR})$ in the deformed nuclei ${ }^{1,2}$ has attracted considerable theoretical and experimental interest. This splitting of the GMR was inferred from the comparison of the relative strengths of the two components of the GR region in the spherical nucleus ${ }^{144} \mathrm{Sm}$ with those in the deformed ${ }^{154} \mathrm{Sm}$ (Ref. 1) and from the systematics of the energy of the GMR in a large number of deformed nuclei. ${ }^{2}$ The observation of two GMR components has also been reported in the actinide nuclei. ${ }^{3}$ The splitting of the GMR has been explained as resulting from the mixing of $L=0$ and $L=2$ oscillations with the onset of deformation which results in a sharing of strength between the GMR and the $K=0$ component of the giant quadrupole resonance (GQR). ${ }^{4-8}$ However, the behavior of these modes in the transitional nuclei has not been understood yet. Although detailed calculations have not become available so far, the model of Kishimoto ${ }^{4-6}$ predicted that in the transitional nucleus ${ }^{148} \mathrm{Sm}$, the $L=0$ and $L=2$ modes will be so mixed that it might be impossible to identify the $L=0$ and $L=2$ contributions to the GR "bump" observed in the inelastic scattering spectrum. This paper reports our measurements on the transitional nuclei ${ }^{148} \mathrm{Sm}$ and ${ }^{146} \mathrm{Nd}$ as well as on the spherical ${ }^{142} \mathrm{Nd}$ and the deformed ${ }^{15} \mathrm{Nd}$.

\section{EXPERIMENTAL PROCEDURE}

Inelastic scattering spectra were measured for $129 \mathrm{MeV}$ $\alpha$ particles, obtained from the Texas A\&M University cyclotron, and incident on enriched, self-supporting, metalfoil targets of ${ }^{148} \mathrm{Sm}$ and ${ }^{142,146,150} \mathrm{Nd}$. The scattered particles were detected in the focal plane of the Enge split-pole spectrograph using a resistive-wire proportional counter.
The experimental techniques including data handling procedures and distorted-wave Born approximation (DWBA) calculations have been described in detail previously. ${ }^{9}$ Special care was taken in the preparation and handling of the targets to minimize contamination; our spectra show no strong peaks attributable to target contaminants that would compromise the peak-fitting procedure. Data were

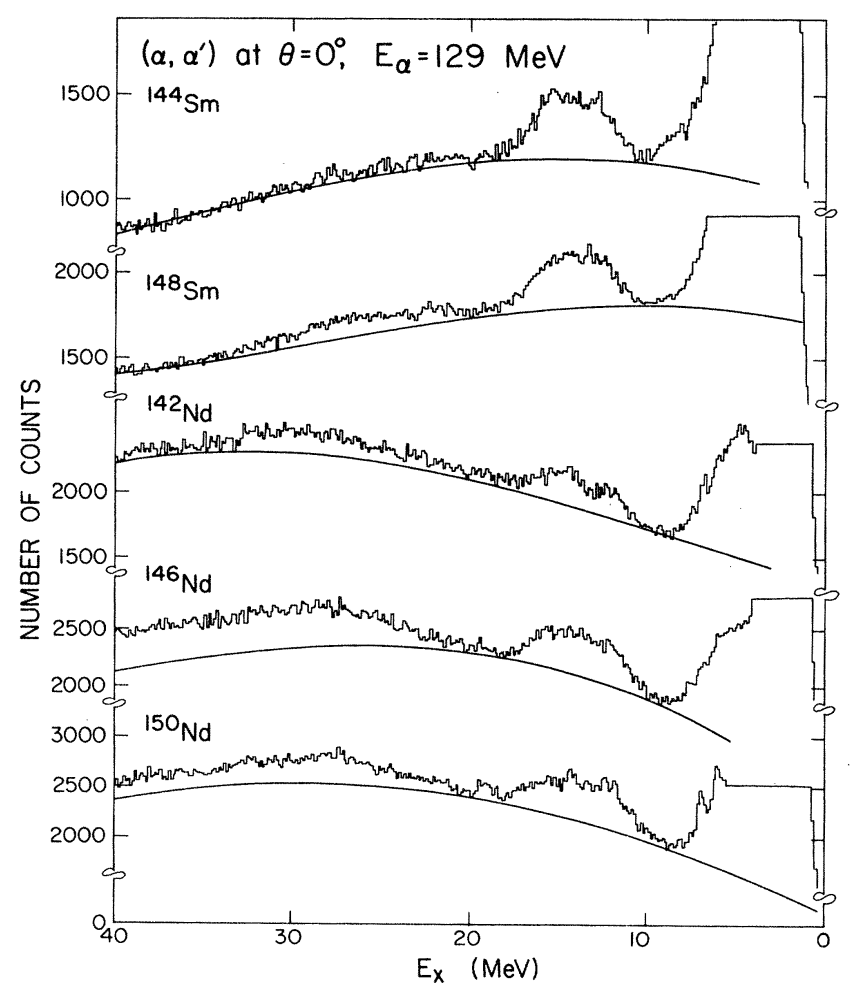

FIG. 1. Inelastic $\alpha$-scattering spectra at $0^{\circ}$ for ${ }^{144} \mathrm{Sm},{ }^{148} \mathrm{Sm}$, ${ }^{142} \mathrm{Nd},{ }^{146} \mathrm{Nd}$, and ${ }^{150} \mathrm{Nd}$. The solid lines show the assumed shape of the background for subtraction. 
TABLE I. Parameters obtained for GR peaks in ${ }^{142,146,150} \mathrm{Nd}$ and ${ }^{148} \mathrm{Sm}$.

\begin{tabular}{lcccc}
\hline \hline Nucleus & $E(\mathrm{MeV})$ & LE peak & & \multicolumn{1}{c}{ HE peak } \\
& & $\Gamma(\mathrm{MeV})$ & \\
${ }^{142} \mathrm{Nd}$ & $12.1 \pm 0.2$ & $2.9 \pm 0.3$ & $14.8 \pm 0.3$ & $3.3 \pm 0.2$ \\
${ }^{146} \mathrm{Nd}$ & $12.2 \pm 0.2$ & $3.3 \pm 0.3$ & $15.1 \pm 0.3$ & $3.5 \pm 0.2$ \\
${ }^{150} \mathrm{Nd}$ & $12.2 \pm 0.2$ & $3.8 \pm 0.3$ & $15.4 \pm 0.3$ & $3.4 \pm 0.2$ \\
$E_{\mathrm{HE}}-E_{\mathrm{LE}}=2.7 \pm 0.4 \mathrm{MeV}$ for ${ }^{142} \mathrm{Nd}$ & & & \\
$E_{\mathrm{HE}}-E_{\mathrm{LE}}=3.2 \pm 0.4 \mathrm{MeV}$ for ${ }^{150} \mathrm{Nd}$ & & & \\
$\Gamma_{\mathrm{LE}}\left({ }^{150} \mathrm{Nd}\right)-\Gamma_{\mathrm{LE}}\left({ }^{142} \mathrm{Nd}\right)=0.9 \pm 0.4 \mathrm{MeV}$ & & & \\
& & & \\
${ }^{148} \mathrm{Sm}$ & $12.1 \pm 0.2$ & $3.1 \pm 0.2$ & $14.6 \pm 0.3$ & $3.4 \pm 0.3$ \\
\hline \hline
\end{tabular}

obtained for all targets at several angles between $0^{\circ}$ and $6^{\circ}$; the $L=0$ and $L=2$ modes exhibit strikingly different angular distributions in this region. An "active collimator" system was employed with the solid-angle-defining slits of the spectrograph to eliminate the slit scattering events and a pulser signal was fed through the data manipulation circuits and the computer to monitor the intrinsic dead time of the system. Concurrent with ${ }^{148} \mathrm{Sm}$, data were also obtained on the spherical nucleus ${ }^{144} \mathrm{Sm}$ to afford a direct comparison.

\section{RESULTS AND DISCUSSION}

Figure 1 shows the "raw" spectra for the aforementioned targets at $0^{\circ}$. The GR regions in the inelastic spectra, after subtraction of a smooth continuum background (the assumed shapes of which are displayed in Fig. 1), were analyzed in the usual way, ${ }^{9}$ assuming two Gaussian components in the GR peak and performing multispectrum fits. The resonance parameters for the two components were extracted and are summarized in Table I. For ${ }^{142} \mathrm{Nd}$ and ${ }^{150} \mathrm{Nd}$, the relative cross section data are presented in Table II for $0^{\circ}$ and $5^{\circ}$, where the relative intensities of GMR are maximal. The angular distributions for the GR components for ${ }^{142,150} \mathrm{Nd}$ are shown in Fig. 2(a).

The splitting of the GMR is evident from Table II: While the total cross section of the low-energy (LE) and high-energy (HE) components is practically the same in ${ }^{142} \mathrm{Nd}$ and ${ }^{150} \mathrm{Nd}$, there is a definite "shift" of strength from the $\mathrm{HE}$ to $\mathrm{LE}$ component in ${ }^{150} \mathrm{Nd}$. The extent of this shift for the Nd nuclei is smaller than that for the Sm nuclei. Nevertheless, in all other relevant respects, viz., the angular distribution of the LE component and the relative energy separation of two components, the $\mathrm{Nd}$ data are qualitatively similar to the Sm data reported in Ref. 1.
The "splitting" of the GMR has been explained in the random-phase approximation (RPA) calculations of Kishimoto, ${ }^{4-6}$ Abgrall et al., ${ }^{7}$ and Zawischa et al. ${ }^{8}$ In a nucleus with a deformed ground state the $L=0$ and $L=2$ oscillations mix, resulting in two $K=0$ states which share the $L=0$ strength between them. In the model of Abgrall et al., ${ }^{7}$ the GQR splits into two components, the axial $\beta$ and nonaxial $\gamma$, where the $K=0$ and 2 components coincide with $\beta$ and $\gamma$, respectively. In the models of Kishimoto ${ }^{4-6}$ and Zawischa et al., ${ }^{8}$ the GQR splits into three components corresponding to $K=0,1$, and 2 . The coupling of $L=0$ and $L=2$ modes leads to a redistribution of $L=0$ and $L=2$ strengths as shown for the model of Abgrall et al. ${ }^{7}$ in Fig. 3 for a hypothetical nucleus with deformation $\epsilon=0.3$. In all three models, the extent of this sharing of strengths depends on the deformation of the nucleus. This feature would explain the smaller amount of "shifted" GMR strength in the Nd nuclei when compared to the Sm nuclei; the deformation $\epsilon$ for ${ }^{150} \mathrm{Nd}$ is 0.236 while that for ${ }^{154} \mathrm{Sm}$ is $0.284 .{ }^{10}$ However, the observed "shift" of strength from GMR to GQR in both cases is larger than that predicted by the theoretical models and this discrepancy between the theoretical and experimental results needs to be further explored.

For the transitional nuclei ${ }^{148} \mathrm{Sm}$ and ${ }^{146} \mathrm{Nd}$, the ratios, $R$, of the cross sections for the high-energy (HE) and lowenergy (LE) components,

$$
R=(d \sigma / d \Omega)_{\mathrm{HE}} /(d \sigma / d \Omega)_{\mathrm{LE}},
$$

were extracted. The angular distribution for this ratio is displayed in Fig. 2(b) and is compared with that for ${ }^{144} \mathrm{Sm}$. Note that for the spherical nucleus ${ }^{144} \mathrm{Sm}$, the HE and LE components correspond to the GMR and GQR, respectively. Also shown in Fig. 2(b) is a DWBA calculation for $R$ for ${ }^{144} \mathrm{Sm}$. While the values of $R$ for ${ }^{144} \mathrm{Sm}$ very closely

TABLE II. GR cross sections relative to $\left(\sigma_{\mathrm{LE}}+\sigma_{\mathrm{HE}}\right)$ in ${ }^{142} \mathrm{Nd}$.

\begin{tabular}{ccccc}
\hline \hline Angle & Nucleus & $\sigma_{\mathrm{LE}}$ & $\sigma_{\mathrm{HE}}$ & $\sigma_{\mathrm{LE}}+\sigma_{\mathrm{HE}}$ \\
\hline $0^{\circ}$ & ${ }^{142} \mathrm{Nd}$ & $0.36 \pm 0.07$ & $0.64 \pm 0.09$ & 1.0 \\
$0^{\circ}$ & ${ }^{150} \mathrm{Nd}^{\mathrm{a}}$ & $0.70 \pm 0.10$ & $0.52 \pm 0.07$ & $1.22 \pm 0.14$ \\
& & $(0.57 \pm 0.08)$ & $(0.43 \pm 0.06)$ & $(1.00 \pm 0.10)$ \\
$5^{\circ}$ & ${ }^{142} \mathrm{Nd}$ & $0.57 \pm 0.06$ & $0.43 \pm 0.06$ & 1.0 \\
$5^{\circ}$ & ${ }^{\circ} \mathrm{Nd}^{\mathrm{a}}$ & $0.82 \pm 0.09$ & $0.29 \pm 0.05$ & $1.11 \pm 0.10$ \\
& & $(0.74 \pm 0.08)$ & $(0.26 \pm 0.05)$ & $(1.00 \pm 0.10)$ \\
\hline \hline
\end{tabular}

a Numbers in parentheses correspond to normalization of $\sigma_{\mathrm{LE}}+\sigma_{\mathrm{HE}}$ to 1.0. 

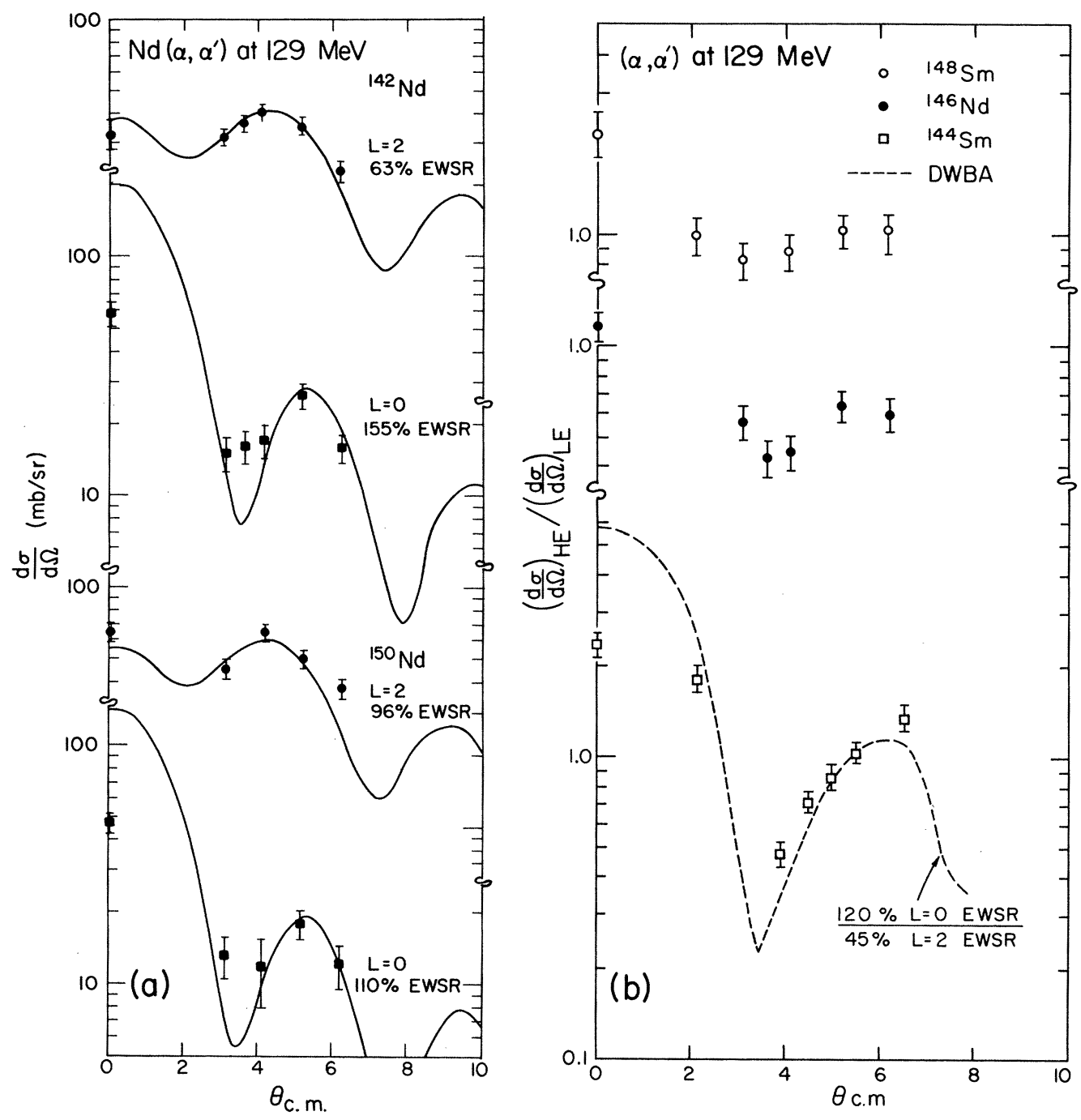

FIG. 2. (a) Angular distribution for the components of the giant resonances in ${ }^{142} \mathrm{Nd}$ and ${ }^{150} \mathrm{Nd}$. The DWBA calculations are shown superimposed. (b) Angular distributions for the ratio $R$, described in the text, for ${ }^{144,148}$ Sm and ${ }^{146}$ Nd. The DWBA calculation for $R$ is shown superimposed on the ${ }^{144} \mathrm{Sm}$ data.

follow the DWBA predictions, they exhibit a strikingly different angular distribution in the case of ${ }^{148} \mathrm{Sm}$. In fact, for ${ }^{148} \mathrm{Sm}$, this ratio is more or less constant over the angular range $2^{\circ}-6^{\circ}$. This feature has been consistently observed in ${ }^{148} \mathrm{Sm}$ in various measurements with different incident $\alpha$ energies $(96,115$, and $129 \mathrm{MeV}$ ) and different targets and clearly shows that the two components of the GR peak in ${ }^{148} \mathrm{Sm}$ exhibit very similar angular distributions over this angular range. In contrast, similar analyses always yield strikingly different angular distributions for the two GR components in the spherical nuclei. ${ }^{1,2,9}$ Furthermore, the angular distributions of both GR components in ${ }^{148} \mathrm{Sm}$ are consistent with a combination of $L=0$ and $L=2$ transfer; unfortunately, though, the errors associated with these measurements prohibit a unique determination of the relative $L=0$ and $L=2$ contributions from the angular distribution data.

Although none of the aforementioned theoretical models dealing with the effects of deformation on collec- tive vibrations has explicitly treated the transition nuclei, certain predictions can be made for ${ }^{148} \mathrm{Sm}$ in the approach developed by Kishimoto. ${ }^{4-6}$ In this model, a rigorous self-consistency is applied which leads to a modification of the usual quadrupole-quadrupole interaction; this approach has successfully explained the broadening of the GQR and the splitting of the GMR in the deformed nuclei. In the transitional nucleus ${ }^{148} \mathrm{Sm}$, the $L=0$ and $L=2$ modes are predicted to be in a phase of extensive mixing which arises from a combination of the splitting of the GMR and successive couplings of the GQR with the low-lying collective $2^{+}$state $\left(E_{x}=0.55 \mathrm{MeV}\right)$ in ${ }^{148} \mathrm{Sm}$. As a result, both components of the GR peak would share the $L=0$ and $L=2$ strengths and hence would show more or less identical angular distributions-a combination of the $L=0$ and $L=2$ angular behaviors. The experimental observations for ${ }^{148} \mathrm{Sm}$ are qualitatively consistent with these predictions. The ${ }^{146} \mathrm{Nd}$ data, however, do not show similar agreement; the observed ratio $R$ for ${ }^{146} \mathrm{Nd}$ is dif- 


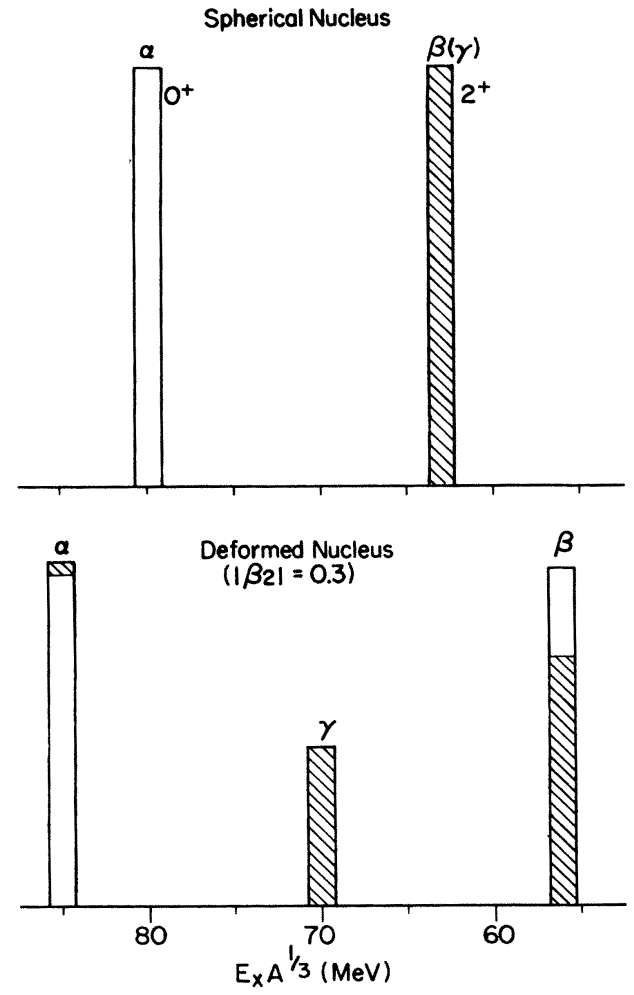

FIG. 3. Schematic strength distribution of the giant resonances in a deformed nucleus according to the model of Abgrall et al. (Ref. 7). $\alpha, \beta$, and $\gamma$ are, respectively, the monopole, axial, and nonaxial components.

ferent from that of ${ }^{148} \mathrm{Sm}$ and, in fact, is more akin to ${ }^{150} \mathrm{Nd}$. This might be attributable to the fact that ${ }^{146} \mathrm{Nd}$ is more deformed than ${ }^{148} \mathrm{Sm}\left(E_{2^{+}}=454 \mathrm{keV}\right.$ for ${ }^{146} \mathrm{Nd}$ compared with $550 \mathrm{keV}$ for ${ }^{148} \mathrm{Sm}$ ) and it is likely that the process of "splitting" of the GMR is already under way.
In a similar vein, an "incomplete" mixing of $L=0$ and $L=2$ modes would explain the increase in the value of $R$ for ${ }^{148} \mathrm{Sm}$ at $0^{\circ}$ since the $L=0$ character of the HE component would most strongly manifest itself at $0^{\circ}$.

The amount of mixing of the $L=0$ and $L=2$ strengths predicted by a simple macroscopic model is, however, much smaller than what is apparent from the experimental data. This is understandable since the validity of a macroscopic model for "transitional" nuclei may not be justified and it might be necessary to employ the effective interactions stated in Ref. 6, which contain more complex coupling terms, for a more reliable calculation. Furthermore, and perhaps most importantly, the inclusion of higher-phonon states as well as coupling to states built on the GMR is expected to enhance the fragmentation of the GQR to higher excitation energies, thus bringing the theoretical results in closer agreement with the experimental observations.

It is clear from the above discussion that a considerable amount of additional theoretical and experimental effort is required to fully understand the behavior of collective modes in the transitional nuclei. It is hoped that the measurements reported herein would encourage further exploration of this important aspect of the collective behavior of nuclei. Measurements on other transitional nuclei, specifically ${ }^{146,150} \mathrm{Sm}$ and ${ }^{148} \mathrm{Nd}$, might be particularly instructive.

\section{ACKNOWLEDGMENTS}

The authors wish to acknowledge the assistance of Dr. John Sullivan and Mr. Mark Parkin in some of these measurements and are grateful to Dr. T. Kishimoto for communicating his theoretical results prior to publication. This work was supported in part by the U.S. Department of Energy, the National Science Foundation, and The Robert A. Welch Foundation.
*Permanent address: Physics Department, University of Notre Dame, Notre Dame, IN 46556.

${ }^{1}$ U. Garg, P. Bogucki, J. D. Bronson, Y.-W. Lui, C. M. Rozsa, and D. H. Youngblood, Phys. Rev. Lett. 45, 1670 (1980).

${ }^{2}$ M. Buenerd, D. Lebrun, Ph. Martin, P. de Saintingnon, and C. Perrin, Phys. Rev. Lett. 45, 1667 (1980).

${ }^{3}$ H. P. Morsch, M. Rogge, P. Turek, C. Mayer-Boricke, and P. Decowski, Phys. Rev. C 25, 2939 (1982).

${ }^{4}$ T. Kishimoto, J. M. Moss, D. H. Youngblood, J. D. Bronson, C. M. Rozsa, D. R. Brown, and A. D. Bacher, Phys. Rev. Lett. $\underline{35}, 552$ (1975).

5T. Kishimoto, in Proceedings of the RCNP International Sym- posium of Highly Excited States in Nuclear Reactions, Osaka, 1980, edited by H. Ikegami and M. Muraoka (RCNP, Osaka University, 1980), p. 145.

${ }^{6}$ T. Kishimoto, see Ref. 5, p. 377; and (unpublished).

${ }^{7}$ Y. Abgrall, B. Morand, E. Caurier, and N. Grammaticos, Nucl. Phys. A346, 431 (1980).

${ }^{8}$ D. Zawischa, J. Speth, and D. Pal, Nucl. Phys. A311, 445 (1978).

${ }^{9}$ C. M. Rozsa, D. H. Youngblood, J. D. Bronson, Y.-W. Lui, and U. Garg, Phys. Rev. C 21, 1252 (1980).

${ }^{10}$ K. E. G. Lobner, M. Vetter, and V. Honig, Nucl. Data Tables A7, 495 (1970). 